\title{
A Complexity Basis for Phenomenology: how information states at criticality offer a new approach to understanding experience of self, being and time
}

\section{by Alex Hankey}

\begin{abstract}
In the late $19^{\text {th }}$ century Husserl studied our internal sense of time passing, maintaining that its deep connections into experience represent prima facie evidence for it as the basis for all investigations in the sciences: Phenomenology was born. Merleau-Ponty focused on perception pointing out that any theory of experience must in accord with established aspects of biology i.e. embodied. Recent analyses suggest that theories of experience require non-reductive, integrative information, together with a specific property connecting them to experience. Here we elucidate a new class of information states with just such properties found at the loci of control of complex biological systems, including nervous systems.
\end{abstract}

Complexity biology concerns states satisfying self-organized criticality. Such states are located at critical instabilities, commonly observed in biological systems, and thought to maximize information diversity and processing, and hence to optimize regulation. Major results for biology follow: why organisms have unusually low entropies; and why they are not merely mechanical. Criticality states form singular self-observing systems, which reduce wave packets by processes of perfect self-observation associated with feedback gain $\mathbf{g = 1}$. Analysis of their information properties leads to identification of a new kind of information state with high levels of internal coherence, and feedback loops integrated into their structure.

The major idea presented here is that the integrated feedback loops are responsible for our 'sense of self', and also the feeling of continuity in our sense of time passing. Long-range internal correlations guarantee a unique kind of non-reductive, integrative information structure enabling such states to naturally support phenomenal experience. Being founded in complexity biology, they are 'embodied'; they also fulfill the statement that 'The self is a process', a singular process.

High internal correlations and René Thom-style catastrophes support non-digital forms of information, gestalt cognition, and information transfer via quantum teleportation. Criticality in complexity biology can 'embody' cognitive states supporting gestalts, and phenomenology's senses of 'self,' time passing, existence and being. 


\section{INTRODUCTION}

The phenomenon of experience with its inner sense of 'self', together with the sense of passing of time, and the sense of existence or being, has been the subject of analysis and speculation since philosophy began. Each of us answers, 'Present!' to a roll-call; we all have a 'sense of our own presence' though we may not know how it arises. The emergence of energy as a fundamental concept in the $19^{\text {th }}$ century and the founding of experimental psychology led to the dismissal of traditional concepts by science at the time. It was said that first psychology lost its soul, then it lost its mind. Whether or not it was in reaction to this, history may not relate, but around that time, the young Edmund Husserl, despite showing promise as a mathematician, decided to switch his field of research to philosophy and started studying with Brentano, whose work ${ }^{1}$ deeply impressed him.

Husserl's mature work focused on the subjective experience of time. Its experience as an internal reality, contrasting with the experience of space as an external reality, has been noted in both East and West for millennia. Husserl made critical use of it in developing Phenomenology, his philosophy of experience, particularly using it in the title of his fundamental work, The Phenomenology of Internal Time Consciousness ${ }^{2}$, which laid the foundation for the development of Phenomenology as a field of study and its spreading throughout the world.

Husserl's student, Martin Heidegger, further developed his teacher's approach, in his fundamental 'Sein und Seit' ${ }^{3}$ - 'Being and Time', but differed from him in his analysis and detailed opinion of the structure of the time concept, developing his own interpretation as 'Dasein'. To most scientists, these ideas remain obscure, almost an anathema. This article presents a new biologically based class of information state, which can form a biophysical basis

for a scientific model of subjective experience, with abilities to support (a) a sense of self, (b) a sense of continuing existence, and therefore (c) senses of both 'being' and 'time': a biophysical model for phenomenology.

These information states occur in complex biological systems at their loci of control. They are therefore serendipitously well placed to give whole organisms the sense of identity and continuing existence, which we humans agree to be the basis for effective interaction with the world of sense perception. They therefore satisfy the basic requirement noted by another famous 
philosopher of Phenomenology, Maurice Merleau-Ponty, who emphasized the need for phenomenal experience to be embodied ${ }^{4}$, i.e. coupled to known biological structures. This in itself raises interesting points, because in the absence of the theory presented in this article, the neural and cognitive sciences usually assume that the only form of information available to the brain is digital, the kind of information exhibited by visual images mapped onto cells in the optical cortex, and similar digital models of information on the surface of the brain, originating in the work of Warren McCullough. ${ }^{5}$

This is probably not true. The brain represents information that way in the cortex before it is cognized in experience. The structure of cortical networks of neurons enables them to support the intricate phenomena associated with complexity biology. Only when transformed by a specialized neural process, mathematically equivalent to a 'linear transform', does information enter conscious experience, and then only in processed form.

As recounted in the next section, loci of control of complex biological systems are states of critical instability, or 'criticality', with properties completely different from ordinary states of matter. This forms the seed for the thesis presented in this article: complex biological systems contain control structures with information states supporting an internal sense of self i.e. of subjectivity, and a continuous sense of existence, or 'being in time'.

Merleau-Ponty's principle of embodiment presents an important condition that any theory of experience must satisfy: it must be consistent with the empirical basis of biology; the theory presented here satisfies it fully. Previous restriction of information theories to digital information, which obviously cannot support a subjective sense, has come to be interpreted as meaning that 'embodiment' restricts biological systems to digital information representations of experience, and that any sense of 'self', or subjectivity, is baseless. Although it has thus come to be interpreted to mean that only the physical body exists, and that all sense of subjectivity is illusory, the approach adopted in this article shows that this is not the case. In complexity biology, organisms can 'embody' Husserl and Heidegger's three phenomenological properties of Self, Being and Time.

Preliminary aspects of this work have been presented to the continuing series of biennial conferences, 'Towards a Science of Consciousness', held at the University of Arizona, Tucson. 
The approach has therefore been more oriented to the kind of questions raised by the organizers of those conferences, such as Bernard Baars, David Chalmers, and Dr Stuart Hameroff. Tucson phenomenology goes beyond philosophy, and attempts to develop scientific theories of how conscious experience is embedded in the brain and nervous system. In this endeavor, it looks to the fundamental work of David Chalmers ${ }^{6}$, who together with his colleague, Jonathan Shear ${ }^{7}$, has distinguished between the 'hard' and 'easy' problems of consciousness. The easy problems, Chalmers stated, consist of questions that can be solved by understanding conventional information processing in the nervous system, such as how memory or emotional states are brought to awareness, difficult though that may be. The 'hard' problem consists of questions clearly not susceptible to explanations in terms of conventional digital-type information processing. ${ }^{6}$ They may include the three phenomenological properties, and how qualitative properties of experience, qualia, such as harmonies, colours, tastes and smells, or even pleasure and pain, take the form they do. As Chalmers states ${ }^{6}$, experience is the hard problem.

Chalmers' achievement included identification of conditions that physical theories of experience must satisfy. These include conditions on the theoretical physics to be used, and are thus connected to my own training as a theoretical physicist. This article's concern is with the physics of information states supporting phenomenal experience. Details of the physics are necessary to show how the proposed information states satisfy properties proposed by Husserl, Heidegger and Merleau-Ponty, and their illustrious predecessors like Descartes, and Immanuel Kant. What of the wealth of earlier, eastern philosophers who perceptively discussed the whole question, often bringing out important highly valuable points of their own, presaging more modern, $20^{\text {th }}$ century versions? A further special issue promises to remedy any such deficiency.

As is obvious to one and all, digital information processing structures do not communicate a sense of their own experience, in the same way that humans can, or animals with whom humans have learned to communicate, including chimps, gorillas, dogs, cats and parrots etc. Anyone who has read, 'The Elephant Whisperer's and its aftermath, or Elsa the Lioness ${ }^{9}$ will realize that selfawareness is far from being limited to the human condition, though verbal language and its use in higher intellectual dialogues, problem solving and political ideologies may be. Humans know what it is 'like' to be human, as Nage ${ }^{10}$ has pointed out. Animals, one suspects, also know what it is like to be their particular species. But, as Penrose ${ }^{11}$ argued, even the world's greatest 
supercomputer cannot be expected to answer the question, 'What is it like to be a computer?' The deficiency, we propose, lies in the kind of information that it is designed to process.

The extent to which this article makes inroads on the 'hard' problem will be discussed at the end, but the results obtained are certainly without precedent and speak for themselves, requiring little justification for the approach adopted. Many contributions to this volume position themselves carefully. The Gare-Kauffman prologue ${ }^{12}$ discussing endophysics and endobiology, for example, represents a collaboration between a philosopher and a scientist, both of whom are leaders in their respective fields. It contains enlightening perspectives on how an expert in phenomenology and a leader in complexity biology can collaborate to illuminate the whole field. The article on 'Situated Phenomenology'13 is precise in justifying its perspective. This article's position is based on the author's academic training and subsequent fields of research, including refereed articles in such areas as critical phenomena ${ }^{14}$, quantum field theory ${ }^{15}$, elementary particle physics ${ }^{16}$, and cosmological nucleosynthesis. ${ }^{17}$ It combines the very different disciplines of quantum theory, control theory, and critical phenomena to perform an unusual analysis of critically unstable states at the heart of regulation in complexity biology - in particular, the properties of their excited states regarded as a new class of information state.

In contrast to the justification expected of a philosopher in adopting the 'position' presented in his perspective on a particular question, a scientific discovery requires no such justification. If worthwhile, the discovery speaks for itself. The problems it solves, major or minor, in its field of concern, justify its perspective. The 'position' of a new approach to interpreting semiotics as a contribution to phenomenology ${ }^{18}$, for example, might require justification, but not a scientific model that can incorporate semiosis, as the one here presented.

On the other hand, a particular school of thought, such as that promoted by Chalmers and his colleagues like Hameroff and Penrose may need positioning. To this author's mind, the Tucson approach is useful, because it defines the physical and information properties to be satisfied by a non-trivial biophysical description of experience. Many people have contributed from many perspectives, and leading mathematical physicists have been, or continue to be involved, including Brian Josephson, Roger Penrose, ECG (George) Sudarshan, and Henry Stapp. Josephson ${ }^{19}$ has suggested that the physics of biology may prove to be more fundamental than the quantum field theories of elementary particle physics. Penrose has made many suggestions. 
First, information structures supporting experience should exhibit high coherence ${ }^{20}$; second the mind's intellectual apprehension of mathematics is capable of making intuitive leaps to the answer transcending any logical reasoning based on ordinary, digital information processing: information processing in the mind almost certainly has no digital analog ${ }^{11}$; third it must be capable of producing orchestrated reduction ${ }^{21}$ of wave packets. This article makes contributions to all these questions.

With scientists of such quality taking active interest in the Tucson approach, little further justification seems necessary. A recent review of that approach by M.I.T. physicist, Max Tegmark $^{22}$ mentions connections between emergence of consciousness and emergence of time, clearly relevant to phenomenology. He suggests that, 'consciousness can be understood as a state of matter... with distinctive information processing abilities', which he calls 'perceptronium'. Here, we follow the spirit of his approach to review the factors now considered essential in any theory of experience, particularly because the theory developed here turns out to satisfy them all, as later sections recount in detail.

One important background perspective is that every culture contains accounts of states of awareness where the information content of experience has been eliminated, and the knower is left in a state where pure self-consciousness alone remains. Such states of 'pure subjective experience', or 'pure consciousness', have been discussed in detail by Shear ${ }^{23}$, who has made their study a large portion of his life's work. Since techniques to train any person, scientist or otherwise, to arrive at clear experiences of such states, are widely available ${ }^{24}$, there are no grounds for denying their existence. Also, Varela and Shear $^{25}$ have justified the acceptance of subjective, first person accounts of experience, so it is reasonable to include them in an article such as this one. The point is that all states of mind with information content are experienced as having the underlying state of pure consciousness at their basis. It is as if the qualia-rich content of experience is superimposed on a content-free state at its basis, similar to the way that quantum excitation content in a quantum field theory is supported by its vacuum state.

Despite being able to enter states where external awareness is not supported, as in deep sleep, or where the sense of identity and objects perceived may seem vague and obscure, as in dreams, consciousness in the waking state constantly maintains a sense of subjective identity, or 'self', and associated senses of 'being and time'. These constitute the essence of experience, and should 
be considered principle attributes of what Damasio ${ }^{26}$ calls, 'The Core Self'. Such a perspective is in distinct contrast to Merleau-Ponty who treated sense-perception as the prime attribute of consciousness $^{4}$, rather than core awareness, of which he apparently knew little.

From this article's perspective, identification of a new form of information supporting subjective experience, David Chalmers's work ${ }^{6,27}$ offers a crucial justification, for he identifies properties that any physical theory should necessarily satisfy. Two concern the internal structure of any theory. He observes, first, that all reductive approaches to explaining experience have failed, and that therefore a (physically speaking) non-reductive theory must be required; and, second, that since known forms of information do not seem to support experience, a special kind of information is needed, one with a second, dual aspect, specifically linking it to subjective experience. As the main sections of this article demonstrate in detail, complexity based information states possess precisely these properties.

Another source of fundamental thinking is Bernard Baars ${ }^{28}$ who, prior to Chalmers's entry into the field, pointed out that experience comprises highly different categories, all of which coexist in a coordinated way. At a party, one can stand balanced on one foot, hold a drink, enjoy the aroma of the food, admire a woman's dress, and hold an animated conversation, all at the same time. Any theory of experience should be able to support such a diversity of inputs and outputs. For this he developed a theory of an overarching structure called the 'Global Workspace of Consciousness'. ${ }^{28}$

Giulio Tononi ${ }^{29}$ similarly emphasizes that the structure of information in experience must be highly integrated, so that different objects in the field of perception can be experienced by the same subject, rather than by a seeming diversity of subjects. Tononi's requirement is not far from the perspective of $\mathrm{Kant}^{30}$, who, to counter the objections from Hume, used the perception of objects as 'wholes' to justify the concept of self. The new information states do indeed support an integrated information theory, in which Kant's point is also upheld.

The work of Penrose, Chalmers, Baars and Tononi all set conditions on the kind of information structure required to support experience. Their requirements are related. Any system

(1) exhibiting sufficient coherence (Penrose ${ }^{11}$ ),

(2) will be non-reductive (Chalmers ${ }^{27}$ ), 
(3) will potentially provide a Global Workspace $\left(\right.$ Baars $\left.^{28}\right)$, and

(4) can only support integrated information $\left(\right.$ Tononi $\left.^{29}\right)$.

The original suggestion that internal coherence is a precondition for consciousness was by Domash $^{31}$, but both his suggestion and Penrose's ${ }^{11}$ were rejected on the grounds that quantum coherence cannot be stabilized at room temperature. This article's solution, critical instability coherence, does not suffer from that drawback.

The closest proposal to that given here has been by Crick and $\mathrm{Koch}^{32}$ who suggested that feedback loops hold the key, but did not make the extension to feedback instabilities. No one else appears to have investigated the idea developed here ${ }^{33}$, feedback instability. Interestingly, Crick and Koch specifically indicated loops formed by thalamo-cortical and cortico-thalamic tracts, for which a suggested application of their critical instabilities ${ }^{34}$ is summarized in the subsection on criticality in signaling pathways.

Here we suggest that deeper properties of neuronal networks in brain cortices must be utilized, their well attested states of criticality. ${ }^{35}$ As detailed in the next section, critical feedback instabilities are rife in biological regulatory systems, for reasons hypothesized, but not yet proved: they appear to maximize information diversity ${ }^{36}$, optimize function and regulation of function. ${ }^{37}$ Any theory based on critical instabilities will be fully 'embodied' in biology, as Merleau-Ponty ${ }^{4}$ required, and Varela and colleagues have explained. ${ }^{38}$

\section{COMPLEXITY BIOLOGY}

Complexity biology has evolved over the past half century, largely due to the impetus given by the work of Stuart Kauffman ${ }^{39}$, and one or two others such as the Danish physicist, Per Bak ${ }^{40,41}$ and US theorist, John H. Holland. ${ }^{42}$ Its significance is only slowly becoming appreciated, and it does not yet have the place in elementary biology textbooks that it undoubtedly deserves. That, however, is slowly beginning to change. In 2014, President of the Royal Society, Sir Paul Nurse wrote in Cel1 ${ }^{43}$, 'For future understanding of biology, we have to get to grips with complexity.' Indeed, there is probably far greater richness lying hidden in complexity than Nurse or even Kauffman have yet begun to suspect. For those not familiar with it, a summary of complexity's essential concepts is now presented, together with a novel, but simple way of estimating their significance. As will be seen, they cast biology in a completely new light. 
Kauffman's original studies ${ }^{39}$ were of patterns of connectivity between loops of genes expressed in genetic networks, identifying which patterns might produce possible kinds of cell. His conclusion was that genetic networks can only produce the kind of pattern of cell types observed in organisms, if each expressed loop of genes coordinates with the expression or repression of an average of two other loops of genes. Smaller coordination values produced uninteresting static possibilities, larger values produced chaos. Genetic networks, Kauffman suggested, function at 'The Edge of Chaos'. This started him on his subsequent half century's investigations of complex biological systems, culminating in his current interests and insights recounted in the central sections of his Prologue to this volume ${ }^{12}$ together with Arran Gare.

Some twenty years after Kauffman's initial contributions, Per Bak and his colleagues were studying unusual fractal distributions of response ${ }^{40}$ found in many physical systems. They came to the conclusion that such distributions would only be possible if the system comes to rest at the edge of instability. The classic example of such instability is a slope to which objects are continually being added ${ }^{44}$, either coming to rest or stimulating the start of avalanches large or small. Examples include cinder cone volcanoes like Japan's Mt Fuji, avalanche slopes of freshly falling snow, piles of sand being freshly sieved on building sites, or the piles of tiny crystals in egg-timers ${ }^{44}$; in all cases, the randomly falling bodies maintain the slope at an almost constant, 'critical', value. Below the critical value, the slope is stable, and the falling bodies add to the pile, increasing the angle of the slope. At the critical value itself, avalanches of unpredictable size take place. The critical value for the slope therefore represents a 'critical instability', with a radical difference between behaviors at smaller values and larger ones. Slopes with angles above the critical value tend to avalanche until the angle of the slope decreases to its critical value, while slopes below the critical value tend not to avalanche until the critical value is reached. Instability dynamics thus spontaneously organize the slope to maintain its critical value; hence the name, 'self-organized criticality'. ${ }^{40}$

'Edge of Chaos' and 'Self-Organized Criticality' are closely connected. Avalanches are chaotic responses, so a system in an apparently stable, or semi-stable, state of self-organized criticality, is literally at 'The Edge of Chaos'. The question then arises, 'What sort of patterns emerge from the chaotic responses produced at criticality?' The answer is the (1/f) distributions of response, which Per Bak's work ${ }^{40}$ originally set out to understand. The term '(1/f) distribution' means that 
the size of the response is inversely proportional to the frequency, $f$, of its occurrence. In models, like those mentioned above, size of response to a fixed stimulus is strictly random and unpredictable, in strong contrast to normal mechanical systems, which produce fixed responses to fixed stimuli.

Complexity biology brings into question whether we should consider biological systems merely 'mechanical' systems. This obviously shocking statement iswell based: mechanical systems are expected to give fixed responses to fixed stimuli, but this property no longer holds for complex biological systems, which give highly variable responses to fixed stimuli. This offers the seed of an idea which will be taken up in the discussion section, in light of all presented results: in light of the ability of complex biological systems to support subjective experience based on the information properties of states of regulation at criticality, we should stop classifying such systems as merely mechanical.

Extensive research has since shown that biological systems often maintain a parameter at a specific value by using the principle of self-organized criticality. ${ }^{45}$ Examples abound, particularly in nervous systems, where neuronal nuclei often give (1/f) distributions of response ${ }^{46}$, indicating that the system's natural state of rest is one of self-organized criticality. It is now apparent that self-organized criticality is found far more widely in biological systems than one would expect by chance, almost universally. The principle seems to have some inherent advantage requiring elucidation.

\section{Postulated Reasons for Self-Organized Criticality}

Implicit in Nurse's urging his fellow biologists to 'get to grips with complexity' ${ }^{43}$ is the message to find substantial reasons why biological systems should adopt the principle so widely. The person one would most expect to have some answer to this question is Stuart Kauffman, for he has thought about the question longer than anyone. His papers contain suggestions, based on computer modeling, that it maximizes information diversity ${ }^{36}$ and information processing. ${ }^{47}$

In my own work, I arrived at the conclusion that, by maintaining switching processes close to criticality, regulatory systems can avoid unwanted switching blockages, and improve sensitivity of their switching processes. ${ }^{37}$ How is that possible, you may ask? 


\section{Criticality in Complexity Biology}

In complex biochemical systems involving biochemical pathways, switching processes such as epigenetic switching of a gene from 'On' to 'Off', or vice-versa, immediately produce large changes in the numbers of molecules in biochemical reactions connected to the enzyme(s) produced from the gene. An abrupt change in the density of a particular chemical in a complex system is analogous to a phase transition in a simple system like pure water, or any other pure chemical. Ergo: switching processes may be modeled by phase transitions.

Abrupt responses would lack sensitivity, however. They would also suffer from difficulties common to all phase transitions ${ }^{48}$, where, rather than changing phase, a system enters a metastable state of the inappropriate phase. Such an eventuality would cause biological systems difficulties. If physical parameters in a cell demand a regulatory response, but no phase change occurs because the system has entered a meta-stable state of the wrong phase, then the regulatory response required for appropriate function has failed, compromising organism health, and possibly decreasing chances of survival.

In contrast, when genetic switching processes are maintained close to, or at the phase transition critical point, two advantages accrue: first, the system will always respond smoothly to demands for more enzyme; second, close to a phase transition critical point, no metastable states are possible $^{48}$, so switching processes cannot be blocked. An example of the first is hormesis ${ }^{49}$, a common phenomenon where a tiny amount of toxin stimulates production of more of the enzyme, the active site of which has been blocked. With regard to the second point, it can be used to construct models of ill-health: when the strain of ongoing function moves the locus of control away from criticality, poor regulatory response to environmental demands becomes a possibility. Failure of required system responses represents a compromise of healthy function ${ }^{37}-$ i.e. a degradation of system 'health'. In this sense, poor regulation represents poor health (correspondingly, improving regulation improves health).

These considerations lead to a very simple suggestion for the reason for the observed ubiquity of self-organized criticality and accompanying (1/f), fractal physiology patterns of response in biological systems of all kinds. Criticality represents a form of optimized function, in particular optimized regulation ${ }^{36}$, which may be used to give a scientific definition of system 'health'. 
How could this have come about in the course of evolution? Here the work of John H. Holland on complex adaptive systems ${ }^{42}$ offers key inputs. Complex adaptive systems continuously explore ways to improve their level of success in their ecological niche, co-evolving along with other niche members as they do so. Any factor that improves function, such as improved regulatory response, will naturally make an organism more successful, and will tend to be slowly 'selected' in succeeding generations. On this basis, states of improved function will slowly manifest. As organisms co-evolve with other niche members, forms of optimized function will act as attractors. If criticality optimizes regulation, self-organized criticality will tend to be adopted as a mode of system operation - almost inevitably.

In biological systems, complex or otherwise, regulation is therefore of extreme importance. Environmental pressures tend to favor organisms that function more efficiently in their niche, particularly ones that are better regulated. One strategy to improve regulation is to improve sensitivity of response to environmental changes. Consider the example of epigenetic switching, changing a gene from being down-regulated to up-regulated, or vice versa. The simple picture of epigenetics is of a gene either being switched On, or switched Of', with an abrupt change from one state to the other represented by a phase transition. The requirement of sensitivity of response suggests that the locus of control of gene expression would be better situated at a place where a smooth transition is possible, suggesting that the phase transition critical point should be the preferred locus of control of the switching process.

\section{Criticality as a Condition on Regulation}

The theory of regulation is due to mathematician Norbert Wiener. ${ }^{50}$ Wiener identified the foundation of regulation as loops of information flow by which a controller receives information and transmits appropriate instructions (also a form of information) back to the system being controlled. Such loops are known as feedback loops. The key quantitative, mathematical, concept in a feedback loop is its feedback gain $\mathbf{g}$ : the ratio of signal strengths for successive passes of a signal round the loop. The gain $\mathbf{g}$ must be less than one, $\mathbf{g}<1$, so that the signal dies away, for patterns of information in the loop to remain stable, otherwise they will grower larger, potentially without limit. As $\mathbf{g}$ approaches $\mathbf{1}$ from below, a form of instability results: critical feedback instability. 
The question then arises, could there be circumstances in which this kind of $\mathbf{g}=\mathbf{1}$, critical instability could play the role of a criticality form of instability in complexity biology? One answer is when the feedback loop is used as a feedforward loop to amplify signals on a signaling pathway. The amplification, $\mathbf{A}$, produced by a feedforward loop is given by

$$
\mathbf{A}=(1 /(1-\mathbf{g}))
$$

This increases without limit as the loop's feedback gain, $\mathbf{g}$, approaches the value 1 from below. When the feedback gain round a loop is close to the value for critical feedback instability, the feedforward loop can make the system far more responsive to small input signals. Is it surprising then, that such loops are found on all biochemical, signaling pathways, exocrine, endocrine and cytokine? Interestingly, the ubiquity of such signaling pathway loops throughout biology was the topic of a previous article by $\mathrm{Nurse}^{51}$, a perspectives contribution to Nature. Although their specific function has, in most cases, not been proved, any cell signaling system could use criticality on such loops to improve responsiveness, i.e. regulatory function. Improving responsiveness will improve regulation and function, and lead to increasing organism success, and self-selection. That is certainly a reasonable starting hypothesis to 'get to grips with criticality'.

The physics of criticality is therefore of fundamental importance to biology. As we shall see, it is fundamental to the new approach to phenomenology suggested in this article. The next sections explain it in more detail, from two perspectives, that of feedback loops and that of phase transitions leading to a new, unexpected aspect of criticality: properties of its information states, analysis of which presents a completely new dimension to the study of information, the new kind of information at the heart of this article.

\section{Criticality on Signaling Pathways}

Signaling pathways present another aspect of regulation where proximity to criticality becomes the preferred state of the system. All complex biological systems contain feedback loops on their signaling pathways, the function of which is apparently not yet understood. ${ }^{51}$ An obvious use would be as feedforward loops to amplify incoming signals and so increase sensitivity of system response. When a loop is operating at a gain $\mathbf{g}$, the ratio of energies of the signal at successive passes round the loop), feedforward amplification is produced by the loop. The feedforward 
amplification is by a factor $\mathbf{f}$ proportional to $(1 /(1-\mathbf{g}))$. In the limit, $\mathbf{g} \rightarrow 1^{-}$, tends to 1 from below, this becomes increasingly large, increasing system sensitivity.

Particular signaling pathways where such loops have been suggested as candidates to explain the presence of subjective awareness are those between the metathalamus and brain cortex ${ }^{32}$, in particular, the lateral geniculate nucleus on the visual pathway and the visual cortex, and the medial geniculate nucleus on the auditory pathway and the auditory cortex. In both cases, the existence of both thalamo-cortical tracts transmitting incoming signals from the thalamus to the cortex, and cortico-thalamic tracts, transmitting feedback signals from the cortex to the thalamus, create feedback loops with the ability to create considerable amplification. Were the gain $\mathbf{g}$ to surpass its critical value, $\mathbf{1}$, an internally generated signal would result, with interesting implications for sense perception. ${ }^{34}$

Long-term practitioners of self-transcending systems of meditation ${ }^{24}$ exhibit unusually low auditory thresholds ${ }^{52}$, accurate measurement often requiring recalibration of audiometers. This can be understood in terms of increased gain round the feedback loops between metathalamic nuclei and sensory cortices. ${ }^{34}$ If the gain $\mathbf{g}$ round the loop on such a pathway should exceed $\mathbf{1}$, a self-generated signal would result. ${ }^{34}$ It is therefore relevant that long-term practitioners of eastern meditation systems report experiences of sound (Aum) and light. These may well constitute evidence for criticality states on the feedback loops concerned.

Such experiences are produced essentially by passive means. The meditation systems advocate staying in states of mental silence, and only returning to practice of the instructed technique when disturbances occur, internal or external. Criticality states on the feedback loops therefore seem to be produced spontaneously by the system dynamics. They would appear to be governed by self-organized criticality.

These feedback loops on the auditory and visual pathways were the focus of Crick and $\mathrm{Koch}^{32}$ in their attempt to explain how consciousness arises within the brain. Our analysis suggests that they did indeed identify crucial components of brain pathways activated by those aiming to experience pure consciousness, but that phenomena associated with such feedback loops are still external to the experience of pure consciousness per se. Rather than supporting consciousness 
itself, they are concerned with refined aspects of sense perception, increasing sensitivity to entire signals, and to specific aspects of signals. Most likely they are responsible for selective attention.

\section{Criticality: The Mechanical Approach}

To understand critical instability, the means by which stability is maintained in physical systems needs to be explained. All stable physical systems undergo mechanical oscillations in response to external disturbances, caused by exchange of various kinds of thermal energy due to impacts of energetic quanta of various kinds, photons, atoms and molecules etc. Such oscillations stabilize the system, oscillating about a well defined mean, and returning it to that state when disturbed. They are called the 'normal modes' of the system, and propagate as waves. Their presence is an absolute requirement for a system's local stability. They are its cause. System instability can then be understood as due to failure of its normal modes.

For example, sound waves stabilize local density in a liquid-gas system, while magnetic spin waves stabilize local magnetic moments in magnetic systems. Instability occurs when the system cannot propagate their stabilizing waves. It can be explained by the system's inability to do so. The physics explanation is that, as the critical instability is approached, the velocity of normal mode waves, $\mathbf{v}_{\mathrm{nm}}$, becomes zero ${ }^{48}, \mathbf{v}_{\mathrm{nm}} \rightarrow 0$.

For example, in a fluid, sound waves stabilize local values of density, and the velocity of sound, $\mathbf{v}_{\mathbf{s}}$, is given in terms of pressure, $\mathrm{P}$, and density, $\rho$, by

$$
\left(\mathbf{v}_{\mathbf{s}}\right)^{2}=(\partial \mathrm{P} / \partial \rho)
$$

At the critical point the graph of pressure against density is flat, so $(\partial \mathrm{P} / \partial \rho)$ is zero (equivalently the compressibility ( $\partial \mathrm{V} / \partial \mathrm{P})$, where $\mathrm{V}$ is the volume of a unit mass, becomes infinite), and sound velocity $\mathbf{v}_{\mathbf{s}}$ becomes zero. Zero velocity sound waves cannot propagate, so they vanish. With the vanishing of stabilizing oscillations, the density becomes unstable, and begins to undergo critical fluctuations driven by the systems' internal energy: the system still possesses the same number of internal degrees of freedom, which support thermal energy and entropy, but its internal dynamics are now of a completely different kind, they are governed by 'critical fluctuations'. ${ }^{48}$

In fluids, the highly fluctuating densities of critical fluctuations extend over scales that include the wave-length of light (microns), so that light cannot propagate smoothly through them. 
Instead, it is scattered in the phenomenon known as 'critical opalescence" ${ }^{48}$, making fluids near their critical points opalescent in appearance, similar to the unusual scattering of light seen in opals. As in variable $(1 / \mathrm{f})$ responses to external stimuli in complexity biology, the right experiments would show critical opalescence to have its scattered light photons distributed according to the (1/f) distributions characteristic of self-organized criticality.

Another approach to explaining the disappearance of normal modes in critical systems is in terms of their quantum properties, because they form a system of quanta: phonons constitute the quantum form of sound waves in a fluid, and magnons the quantum form of magnetic spin waves in a magnet. System normal modes can be quantized, and form quantum systems when forces between particles obey Hooke's law with its parabolic form of potential energy. Energy levels are then equally spaced and can be considered to be occupied by a set of equal energy quanta (in practice this is a little more complicated, but the end result is the same).

Local structures of interparticle forces in terms of their potential energies offer a second kind of explanation for normal mode failure, equivalent to the first, also useful to the theme. In normal states of a fluid, interparticle attractions combine with core repulsion to produce an average interparticle potential with a deep minimum, keeping particles in the liquid close together, but insufficient to do so at higher energies in the gas, allowing them to remain far apart. At the critical instability, these tendencies balance, the effective potential develops a flat minimum.

A flat minimum effectively makes the fluid infinitely compressible, $(\partial \mathrm{V} / \partial \mathrm{P}) \rightarrow \infty$., as stated previously, implying that no waves can propagate. However, a second way of looking at the situation is to observe that with a non-parabolic effective potential, energy levels are no longer equally spaced, and cannot be considered to be populated by a set of equal energy quanta. No quantum field is possible. Instability can now be attributed to absence of a stabilizing quantum field.

Hooke's law (and its analogues for various systems) and finite values of compressibility (and its related analogues) are closely related. Despite looking so different, these two ways of explaining how a system becomes unstable are equivalent. But Hooke's law has provided an interesting insight into instability at a microscopic level: failure of system quantizability, which is further developed in the Results section A. 


\section{Properties of Criticality States 1: General Properties}

Two seemingly different ways of considering criticality have been presented, either in terms of switching processes considered as phase transitions, or as critical instabilities on feedback loops, when the gain $\mathbf{g}$ approaches unity, and the system enters the region of a feedback / feedforward instability. The first approach allows criticality states to be compared to critical instabilities at the end points of a line of phase transitions for increasing temperature. This parallel is useful for deriving thermodynamic properties, such as showing how their physics incorporates high levels of long range correlations, making them non-reductive in a new way (Results Section B). The second is useful to derive information properties, demonstrating that they carry Chalmers' dual aspect, central to this article (Results Section C), and how they can conduct Penrose's orchestrated reduction, $\mathbf{O R}$, of wave packets (Results Section A).

First, criticality in terms of phase transitions: below some 'critical temperature' $\mathbf{T}_{\mathbf{c}}$, two phases can coexist, connected by a phase transition, while above the critical temperature, only a single phase exists, so no phase transition is possible. Examples of this kind of behavior, include the liquid-gas and ferromagnetic phase transitions. The first ends in a liquid-gas critical-point for which a theory, still taught in high-school physics, was developed in the $19^{\text {th }}$ century by Van der Waals. The second ends in a ferromagnetic critical point, studied by Pierre Curie (husband of Marie Curie) for whom its critical temperature, the Curie temperature, is named. Above the Curie point, ferromagnets usually become paramagnetic.

As the end point of a line of phase transitions is approached, the properties of the two phases become more and more similar, until transitions between the two can eventually be brought about by thermal fluctuations: the so-called 'critical region'. In this regime, the material cannot decide which phase it should be, and it begins to fluctuate between the two possible states, generating 'critical fluctuations', which dominate system dynamics, and determine properties of the material.

Phase transitions are characterized by a discontinuous change in a variable, known as the system 'order parameter'. In liquid-gas phase transitions, density changes discontinuously from high density in the liquid, to low density in the gas; it constitutes the order parameter. Ferromagnets, show relatively abrupt changes in internal magnetization as magnetic field direction is reversed at zero magnetic field. As the critical point is approached the discontinuous change becomes 
smaller, eventually becoming smooth. In gas-liquid critical points at the critical values $\mathrm{P}_{\mathbf{C}}$ and $\mathrm{V}_{\mathrm{C}}$, the slope of the curve of pressure, $\mathrm{P}$, against volume, $\mathrm{V}$, (negative for an ideal gas, $\mathrm{PV}=\mathrm{RT}$ ) becomes flat, $(\partial \mathrm{P} / \partial \mathrm{V})_{\mathrm{T}}=0$, where the subscript $\mathrm{T}$ indicates that the temperature is held constant, in this case at $\mathrm{T}=\mathrm{T}_{\mathbf{C}}$. The velocity of sound in the fluid is characterized by $(\partial \mathrm{P} / \partial \mathrm{V})$, so when this becomes zero, sound waves cannot propagate. They cannot function, and therefore cannot fulfill their normal role in the fluid (i.e. 'liquid' or 'gas'), which is to maintain the local stability of a given unit volume of the fluid. The density of the fluid therefore becomes unstable, exhibiting large scale fluctuations - critical point fluctuations. The phase transition approach therefore corroborates the mechanical approach to explaining instability.

In general, values of the order parameter in different phases are stabilized by specific kinds of quanta, or quantum field: sound waves, or phonons, in a liquid or gas, and magnetic spin waves, known as magnons, in magnetic systems. As the critical point is approached the value of the order parameter becomes unstable and begins to fluctuate violently; it is no longer well-defined. Large fluctuations in local values of the order parameter constitute the critical point fluctuations.

Mechanical systems are expected to have well defined properties, and although seemingly mechanical reasons have been given for why a critical system has become unstable, namely the velocity of the stabilizing quanta, $\mathrm{v}_{\mathrm{q}}$, has become zero, $\mathrm{v}_{\text {qcrit }} \rightarrow 0$, the system now fails to behave like a mechanical system, in that a fixed stimulus no longer gives a fixed response. In the case of liquid-gas critical points, their highly irregular scattering of light called 'critical opalescence' was known in the $19^{\text {th }}$ century. Point: unlike mechanical systems which give fixed responses to fixed stimuli, and behave predictably, critical systems give highly variable responses to fixed stimuli, continuously varying in time, and in the example quoted, giving an opalescent appearance to scattered light.

These preliminary observations on the effectively non-mechanical nature of critically unstable systems are a double edged sword. They imply that biological systems at criticality should not be considered mere mechanisms. Indeed, fractal physiology ${ }^{53}$ has documented huge numbers of systems where system response to stimuli evokes (1/f) response distributions, which are therefore not fixed. But no one seems to have pointed out that this simple fact denies physiological systems the purely mechanical nature that has always been attributed to them. 
But who has had the courage to ask the question, 'Are there circumstances when a physiological system might not be considered a purely mechanical system?' The above answer seems (to this author at least) undeniable: 'When the system is at criticality!' This means that no well functioning complex biological system should be considered a 'mere mechanism' (as all traditionally have been), because most if not all properly functioning physiological systems are regulated from loci of control at criticality. ${ }^{37}$

The second edge to the sword is that, if as this paper proposes, all states of subjective experience are founded on systems at criticality, then no psychological test should be designed around the assumption that it should yield fixed answers from the same person, nor that the variability in the answers should obey normal distributions - they should obey (1/f) distributions and statistical analysis should use the Sankaran index, not normal distributions and variances. In fact the basic assumptions of behavioral psychology, that the mind is merely a mechanical system, obeying laws of fixed responses to fixed stimuli, become patently false.

\section{RESULTS}

\section{A. Criticality and OR produced by Exact Self-Observation}

As we have seen above, from the perspective of quantum theory, the normal mode oscillations responsible for system stability constitute a system of quantum fields in the medium concerned phonons in liquid-gas systems, and magnons in magnetic systems. System stability depends on their presence, and their correct function. Should they disappear, stability cannot obtain. It turns out that it is possible to explain the disappearance of the system of normal mode quantum fields at critical instabilities purely in terms of feedback. When this is done, the criticality condition is found to result in annihilation of all normal mode quantum fields - in other words criticality produces Penrose's so-called 'Orchestrated Reduction' (OR) of all normal mode wave packets.

This new approach reasons that a loop with feedback gain, $\mathbf{g}=\mathbf{1}$, constitutes a perfectly selfobserving system, and attributes disappearance of the normal mode quantum fields to the state of perfect self-observation. This means that the new reason for the disappearance of the system of stabilizing quanta is particular to quantum theory, and can be formulated without reference to classical physics i.e. mechanics, as was done in the last section. 
Let us therefore consider a control system with a feedback loop, as the feedback approaches its critical value, i.e. the gain $\mathbf{g}$ approaches $\mathbf{g}=1^{-}$from below. Clearly, information flowing round a feedback loop means that each element of the loop is receiving information about itself. Not exactly the information that left that element on its path round the loop, as long as $\mathbf{g}<1$; but as the gain $\mathbf{g}$ approaches $\mathbf{g} \rightarrow 1^{-}$, the information returning to the chosen point in the loop becomes more and more exactly the information, which left it. This means that, as the limit $\mathbf{g}=1$ is attained, the energy and information returning to that element of the system becomes exactly the same as the energy and information that left it on the previous pass. This idea has a vitally important consequence: at criticality a feedback loop can be considered to be making a very special kind of observation of itself, an absolutely exact self-observation. Away from criticality, for $\mathbf{g}<\mathbf{1}$, the system is also self-observing, but it is not exactly self-observing. At criticality, on the other hand, it is exactly and precisely self-observing, quantitatively speaking. Such a system may be termed an 'exactly self-observing system'.

What could be special about such a condition? Enter one of the two fundamental laws of quantum theory as given in its most rigorous formulations: wave functions, or packets of wave functions, or quantum fields, can undergo two fundamental kinds of change, Types I and II:

- First, Type I change, which is discontinuous, when they are observed: 'wave functions collapse'; 'wave packets, mixtures of wave functions, are reduced'; 'quantum fields are annihilated'.

- Second, Type II, continuous change: wave functions, wave packets, and quantum fields, evolve in time according to their equations of continuity, such as those due to Schrodinger, Dirac, or various quantum field theories.

The point we are making is that, an act of exact observation in a feedback loop constitutes a Type I quantum process, and will therefore annihilate all quantum fields in the system including those of the stabilizing normal modes.

Evidently, normal mode quanta in feedback loops experience this condition in modified form. They are not annihilated when feedback gain, $\mathbf{g}$, is far from unity: $\mathbf{g}<\mathbf{1}$. However at $\mathbf{g}=1$, when all energy and information pass round the feedback loop completely unchanged, it seems entirely reasonable to propose that the state of the system has created a sufficiently exact act of 
observation to annihilate all quantum fields within the system, and that this represents a purely quantum explanation for why the system has become unstable, without reference to mechanics: Information arriving at each point in the system is exactly the same as the information which departed. Regarded as an information transmitting system, a feedback loop with $\mathbf{g}=1$ is undergoing a process of perfect self-observation. According to the stated law of quantum theory, such a process of exact or perfect self-observation will destroy all quanta within the system.

To summarize: as a feedback loop's feedback gain, $\mathbf{g}$, approaches $\mathbf{g}=1^{-}$, the entire feedback loop becomes subject to a process of exact self-observation; normal mode quanta are annihilated; they can no longer exist in the system, and with their disappearance, instability results. This presents a completely new, purely quantum, explanation for feedback instability, which can immediately be taken as one of its fundamental properties.

What is important in the current context is that this explanation for instability offers a radical new explanation for Penrose's orchestrated reduction (OR) of wave packets. ${ }^{21}$ Penrose realized that reduction of quantum wave packets requires a highly non-linear theory, and, being expert in quantum approaches to gravity, he knew that they offer non-linearities. His mathematical physics is unquestioned, but many find the source of his explanation far-fetched.

Feedback loops are classic examples of non-linear processes. At feedback instability, their physics is entirely non-linear. They offer a highly appropriate source of non-linear physics to explain orchestrated reduction (OR) of quantum wave packets in biological systems. Critical feedback instabilities with their evident property of perfect self-observation round the loop constitute singular self-observing systems. The process of perfect self-observation at $\mathbf{g}=\mathbf{1}$, collapses all wave functions, reduces all wave packets, annihilating all normal mode quanta. Resorting to quantum gravity is not needed. Non-linearity at criticality in feedback physics as exemplified in complexity biology is quite sufficient.

This analysis of feedback loop criticality can be applied to critical instabilities in any system. A fundamental characteristic of instability in all critical systems, including the thermodynamic examples in fluids and magnets discussed in the previous section is non-linearity. All such critical systems are par example non-linear systems. Any critical instability can be similarly understood as a physical condition where the mathematics of the system is no longer linear, but 
has become non-linear: completely self-interacting i.e. perfectly self-observing. Such systems are also effectively in states of perfect self-observation and normal mode quantum fields responsible for maintaining the system's local stability are destroyed by the non-linearity. As above, the accepted law of quantum theory that acts of observation 'collapse wave functions' then applies: the non-linearity reduces all quantum wave-packets, destroying all quantum fields in the system. This suggests a new law of physics: pure non-linearity annihilates quantum fields.

Conclusion: in a perfectly self-observing system, stabilizing quantum fields are annihilated. Equivalently, non-linearity in any essentially non-linear system effectively results in a process of self-observation, removing stabilizing quantum fields, and producing instability.

\section{B. Information Properties of Criticality States}

That criticality states possess information properties may not seem obvious, but a general line of reasoning based on entropy shows that they must. Information and entropy are opposite ${ }^{54}$, information being negative entropy. Every excitation in material systems contributes to system entropy and can be assigned an information value. Since criticality states possess definite values of entropy, they can also be assigned a corresponding measure of information. Normally, in microscopic systems this is measured in terms of quantum information, but as we have seen, criticality states are not quantum systems, so the theory of quantum information does not apply. Systems at criticality possess a completely different kind of information. This section and the next elucidate its structure, one which is so different as to be completely new, requiring development in stages: first, in this section, considering critical correlations and their consequences.

B1. Correlations ${ }^{19}$ : As explained previously, critical instabilities possess high levels of long range internal correlations. Under normal conditions, the degree of correlation $\mathrm{C}(\mathbf{r})$ between system elements separated by a distance $\mathbf{r}$ varies as

$$
\begin{aligned}
& \mathrm{C}(\mathbf{r}) \propto \mathbf{r}^{-(\mathrm{d}-2+\eta)} \operatorname{Exp}(-\mathbf{r} / \xi) \\
& =\mathbf{r}^{-(\mathrm{d}-2+\eta)} \text { when } \xi \rightarrow \infty
\end{aligned}
$$

where $\eta$ is a critical exponent. This decreases exponentially with $\mathbf{r} / \xi$, where $\xi$ is the 'range' of the correlations. ${ }^{48}$ As the critical point is approached, $\xi$ tends to infinity, the range of correlations becomes infinite. Correlations become long range, every system element is correlated with every 
other element according to the power law, $\mathbf{r}^{-(\mathrm{d}-2+\eta)}$, to an extent not present in ordinary quantum systems i.e. microscopic systems not at critical points. (This represents a different kind of correlation from those in systems of identical particles, because it is not just particles that are correlated but their dynamics.) Critical correlations 'span' the entire system; no system element is left out; no pair of system elements can be considered uncorrelated.

Since they represent a form of internal order, critical instability correlations lower system entropy, a property easily demonstrated from the mathematical form of entropy, $S=-\operatorname{Tr}\{\rho \ln \rho\}$. Two important points follow: (a) correlation negative entropy provides a measure of negentropy information in systems at criticality, a property to which we shall return later; (b) correlations may be able to explain anomalously low entropies ${ }^{55}$ observed in living organisms.

Many years ago two physicists postulated that states of experience should contain high levels of internal correlations, Domash ${ }^{31}$ in public lectures in the 1970's, and Penrose ${ }^{20}$ in the 1980's. Their ideas were rejected on the grounds that long range quantum correlations cannot exist at room temperature. Naturally high levels of quantum correlations are a feature of certain special kinds of low temperature quantum system such as superconductors and superfluids, and depend on low temperatures $\left(-260\right.$ to $\left.-270^{\circ} \mathrm{C}\right)$ for their existence. Being due to system instability, critical point correlations are distinct from them. Unlike low temperature quantum correlations, they are generated by instability and are necessarily stable at temperatures at which instability occurs. The difficulty is overcome. The Domash-Penrose hypothesis is supported.

Low organism entropies ${ }^{55}$ : in the 1960 's, many organisms were observed to have anomalously low entropy levels, but the phenomenon was never understood and seems to have been virtually forgotten. The lowering of entropy at criticality in phase transitions, which can be attributed to the long range correlations and high internal coherence at criticality offers a possible qualitative explanation: organism entropy is decreased by criticality states' internal coherence, a point of significance, not yet stated in complexity biology.

B2. Non-reductive ${ }^{27}$ : Correlations between system states destroy their independent existence. Quantum correlations, and other properties of quantum systems compromise their reducibility. However, critical correlations are so extended, and so inclusive, that they create macroscopic levels of system integration that quantum systems do not exhibit. Long-range correlations make 
systems at criticality so highly correlated as to be non-reductiveto a new level, a key property for subsections B3 and B4.

This can be illustrated as follows, quantum physics expresses states of multicomponent systems as sums and products of states of system components. A system with two components represented by $\psi_{1}$ and $\psi_{2}$ might in some specific case be represented by $\Psi_{12}=\psi_{1} \psi_{2}$. However many components a quantum system may have, its states can be constructed out of sums of products of fields representing those component, is expressible in terms of them. States of quantum systems may be said to be constructible out of its components, and in that limited sense, reducible to them. Mathematically, states of a quantum system form a special kind of vector space known as a Hilbert space, members of which can be expressed as a linear combination of its basis vectors, and in that sense are 'reducible' to them.

At a critical point this is no longer true. System states cannot be expressed as sums and products of states of individual elements within the system. They constitute mixtures of an altogether different level of complexity; Hilbert space basis vectors are no longer available; mathematically they form Banach spaces, rather than Hilbert spaces, and are irreducible to a new degree.

B3. Integrated Information ${ }^{29}$ : In quantum theory wave functions like $\psi_{1}$ and $\psi_{2}$ represent information states. ${ }^{56}$ Information in a multicomponent system equals the sum of the information contained in its component systems. In quantum theory, correlations reduce the amount of entropy in systems containing two or more sources, and so increase the information content to yield a picture of a single integrated system. When correlations in a complex quantum system are high enough, they integrate information about its subsystems into a single picture.

At instabilities, the high levels of long range correlations automatically mean that information content of all their various component structures are correlated: the result is an integrated information theory, no special conditions need be invoked to achieve it. Tononi's proposal ${ }^{29}$ is automatically realized.

B4. Global Workspace ${ }^{28}$ : Baars' concept of a global workspace is related to integrated information, but not limited to a single sense, or a single kind of activity. Once again, the high levels of correlation that exist between different, widely separated components of a system at criticality result in their forming a single overarching structure. 
In this way, the long-range, system spanning correlations in criticality physics enable the system to support an overall information space, in which to represent activities of all elements perceived in external reality. Such correlations can unify different channels of perception, thought and feeling into a single perceptual and felt reality. Integrated overall information states register all channels in the mind simultaneously in an overall Global Workspace for Consciousness.

The above properties of criticality based information states, B1-B4, are summarized in Table 2. They show how long range criticality correlations endow critical instabilities with the properties hypothesized for states of conscious experience by recent philosophers and scientists. Next we consider the structure of information at criticality.

\section{Structure of Information in Criticality States}

This section contains the key derivation of the whole paper: Chalmers' second requirement ${ }^{6,27}$, dual aspect information pertaining to 'self', is demonstrated for critical instability excitations. From the new kind of information, experience information, depicted in Figure 1, we are able to derive the three properties proposed by phenomenology's founders, Husserl and Heidegger. The dual aspect is the most important property developed here because no information state currently being used by those in the Tucson approach to phenomenology has a dual aspect. Hameroff and Penrose assume that it is sufficient to use quantum information, which they propose for the proteins in cells' cytoskeletons. That may well be appropriate for information in memory waiting for retrieval, but is not appropriate for information in cognitive states.

The easiest way to establish a dual aspect for the structure of information states at criticality is to use fluid flow vectors in viscous systems as an illustrative example. In fluids, flow vectors represent a kind of information, so fluid flow vectors are analogous to information vectors. At the boundary between smooth laminar flow and turbulence, where the Reynolds number becomes critical, they exhibit instabilities. Turbulence consists of vortices, vector loops. At the critical instability leading to turbulence, vortices want to form, but cannot quite do so: each flow vector $<=====$ may be considered to have one or more infinitesimal vector loops attached to it

$<=====\mathbf{O}$, each infinitesimally altering the vector's direction: the flow vectors thus become unstable, and consist of mixtures of vector states. 
These properties carry over into information properties of quantum fields of normal modes as a critical point is approached: the quantum field's quantum information vectors gain similar vortex-like loops at criticality, i.e. they become quantum information mixtures, held together by infinitesimal information loops. The information vectors have assumed dramatically altered properties: as depicted in Figure 1, they have become vector mixtures with infinitesimal attached information loops, a vector bundle $<=====$ with an added information loop: $\mathbf{O}$. The effect of the state of perfect self-observation of the critical, $\mathbf{g}=\mathbf{1}$, feedback loop is to incorporate itself into the structure of the information state itself to yield, $<=====\mathbf{O}$, a new kind of information state.

\section{Figure 1: The Structure of Information States at Criticality}

$$
<====+\mathbf{O} \rightarrow<=====\mathbf{O}
$$

Figure 1 Caption: Figure 1 depicts the information properties of excited states of systems at criticality. They exhibit two aspects, an irreducible vector mixture representing information content, and a 'dual aspect' consisting of a loop of information flow, with the property of perfect self-observation.

The proposed $\angle=======\mathbf{O}$ structure is unique to criticality states, and yields an entirely new kind of information theory. Its information structure is completely different from, and unrelated to, the digital information that we are used to in information technology.

But what kind of information might the new, $<=======\mathbf{O}$, structure represent? What function might the loop fulfil? We propose that the effect of the loop is 'to register the state's own existence'. As such it may begin to explain an essential aspect of experience, one that no previous mathematical model has been able to attempt: the sense of self that accompanies all experience. We propose that this property is not merely analogous to a person 'being aware of their own presence' throughout experience of waking state activity, like sense perception, rather the newly identified loop within the structure of the information states represents the mechanism by which self-awareness is maintained during perception, thought, decision making etc.

Note however, it is important to realise that the proposed connection between the loop in the information state and the sense of self in awareness is an hypothesis. The existence of the attached loop in the information states is not in question. Rather its application to describing experience requires an intuitive leap, which forms the hypothesis: the information loop transforms the information state from a merely objective state of information into one of 
subjective experience $e^{33}$, i.e. it constitutes a dual aspect transforming the state's information content into an act of phenomenal experience.

If this hypothesis is valid, the loop represents the source of subjectivity in biological information states used to support experience. In support of this, note that reducing the state's information content to zero reduces the state to one of pure self-knowledge - the traditional definition of the word 'consciousness' in most mid-20 $0^{\text {th }}$ century dictionaries. To expand this point, the model $<======\mathbf{O}$, represents information in experience, or 'experience information'. Of this structure, the loop by itself, $<\mathbf{O}$, represents a state of pure self-knowledge, i.e. the 'self', on top of which any information content from sense perception or thought is actively super-imposed. In the proposed model of experience, the 'self' is therefore represented by a process, a singular loop of information flow, a singular process. The special significance of this here is that Denis Noble has proposed as one of his 'Principles of Systems Biology' that 'the self is a process'. ${ }^{57}$ The model qualifies this to: the 'self' is a singular process, an information loop at criticality.

The means by which such a 'self' registers information is very subtle and may not seem obvious. What the model offers is a set of states with various degrees of excitation of different kinds, analogous to, but different from, a set of excited states in a quantum field theory. The state of least excitation, analogous to a quantum vacuum state (but with additional properties) is the state of pure self-awareness, $<\mathbf{O}$. Its excited states, $<======\mathbf{O}$, represent states of awareness with information content from sources such as sense perception, treated by Merleau-Ponty. By integrating information from thought, memory or perception, $<=======$, into its foundational structure, $<\mathbf{O}$, the information becomes registered in experience as $<======\mathbf{O}$. In eastern thought, it is sometimes stated that, 'the self takes on the form of experience-content', a statement that supports the picture given by $<\mathbf{O} \rightarrow<======\mathbf{O}$ as a model for registration of information in experience by means of pure self-awareness gaining information content.

A singular process, $<\mathbf{O}$, is not an ordinary process. The model endows the 'self' with properties different from any hitherto conceived possible in physical, chemical or biological sciences. The proposed structure of information states at criticality in Figure 1 represents the possibility of a radical new departure in scientific thought. 
To those who find the hypothesis acceptable, or who are inclined to examine it further (a matter of personal taste or judgment), a whole new field may become describable by scientific theory, and eventually a candidate for inclusion in scientific knowledge. The hypothesis thus represents an induction of the kind needed to open up a new field of science; without which no radically new field of science can emerge in the possible scheme of intellectual things, and maybe become established. The question is not whether, on seeing it for the first time, it seems logical, it cannot, for logic is always merely the handmaid of deductive reasoning. The question is rather whether it fits, whether in some way it 'feels right' - whether the new pattern that it offers, provides a beautiful enough fit to the body of phenomena laid out for explanation by, or inclusion in, the overall theory. That it may offer a means to represent a mathematical model of self-knowledge and experience is a small first step

Now consider the other two phenomenological properties ${ }^{2,3}$ : the inherent sense of continuity of time passing, of 'Being in Time'; the inherence of time in experience. The model supports this, because circulation of information round the loop is not a thing, rather it is a process. Again, it is an intuitive step to make the hypothesis that such a loop process can give rise to an internal sense of continuity interpolating between, and connecting successive events that come to experience. Once this is accepted, the model is seen to give rise to the sense of continuity in experience Being in Time - in addition to a sense of self. In other words, the model seems to correctly predict that our 'internal time consciousness"' is inherently embedded in our sense of 'self'.

Time is an intrinsic aspect of self-awareness. Time is not discontinuously experienced, as it would have to be, if only consciously experienced events were able to effect its registration, as in most current neurophysiological models. The apparent continuity of our subjective experience of time then becomes evidence in favour of the model, subliminal registration of information of short duration as in now illegal subliminal advertising, notwithstanding.

The sense of 'self', and the sense of the passing of time, combine to yield a 'sense of our own presence', which can also be taken as an inherent aspect of experience. In support of this, remember that when our name was called out in a roll call in class at school and we replied, 'Present!', our response affirmed our 'sense of our own presence' in class. The connection between that and being present in the sense of being here, now, i.e. 'being in time', is an inherent 
aspect of our subjectivity. It is remarkable that the 'experience information' model seems to offer a theoretical basis for how this happens. The inherent loop seems to 'fit' in many ways.

In summary: At criticality, states of materials are unrecognizably transformed. In many cases they do not even appear to be similar to those of the underlying material. They exhibit completely different physics from that of the original stable matter. Phenomenology and modern consciousness studies both identify properties of 'experience', which differ from those of the material world of perception. One possible perspective is that the two are so different, that two entirely different kinds of physical theory should be used to describe them. The physics of instability, critical phenomena, and the physics of stability, whether classical or quantum physics, are sufficiently different to fulfill this. The physics of stability is supported by normal mode quantum fields, which represent the defining aspect of matter as we know it. The physics of instability, of criticality in biology and singularity in the mathematics of control theory, when understood in terms of singular information states with inherent feedback loops attached, may be able to account for many different properties of experience - of phenomenology.

\section{Information Measures appropriate for Criticality States and their properties}

D1. Negative Entropy of Correlations: Any information state must be assigned an appropriate measure of information. Under B1 in Part B, entropy reduction attributable to correlations was noted as an appropriate measure of information since information represents order, while entropy represents disorder. This implies that information in criticality states is somehow embedded in their long-range correlations, which provide a measure for the information concerned.

This seemingly simple answer only raises further questions: Does information associated with the internal correlations of long-range coherence possess a digital representation? How can it be used to perform calculations? How can information about the external environment be encoded in it? These questions are answered in parts D2-4.

D2. No Digital Representation ${ }^{11}$ : If information is embedded in critical fluctuations, it must be completely distinct from the usual kind of information that we are used to in modern information technology, the digital information ${ }^{58}$ conceived by Claude Shannon. Digital information is represented in materially fixed structures, as magnetization of magnetic domains in hard disks, or as the switching states of logic circuits in microchips. In either case, a material structure that can 
be switched between two states is employed, one state representing a ' 0 ', while the other state represents a ' 1 '.

In the case of critical correlations, no such rigid structures exist, so how could digital information be embedded? Although a negative entropy information measure establishes a digital equivalent for the information, it does not mean that the information is in digital form. Rather the opposite. Being non-digital, information embedded in the system is of a completely different kind.

Penrose was the first scientist to famously question whether information in consciousness obeys the usual laws of information theory that limit most applications in information technology. In 'The Emperor's New Mind'11 he famously concluded that it does not, but was unable to prove his case sufficiently well for a largely hostile scientific community to accept it. He did not explicitly offer any alternative, like the criticality information states suggested here.

Criticality states' ability to support information as coherence information embedded in their long range internal correlations gives a first clue to constructing an argument that establishes Penrose's thesis directly from a model of conscious experience. The hypothesized model of experience does not involve digital information, but information of a very different kind. As shown in B3, its high levels of internal coherence make it a possible form of integrated information, holding together the perception of many objects into a single state of awareness. This suggests that it also supports a completely different kind of representation of objects of perception, where all parts of an object are integrated into perception of the object as a whole, a gestalt.

That we apprehend objects as wholes was first pointed out by Immanuel Kant in his Critique of Pure Reason $^{30}$, which uses the idea as evidence for the existence of the self. In a deeply perceptive argument that was found very compelling, Kant suggested that the role of the 'self' was to hold together the various perceptions of different parts of an object, and to synthesize them into a whole. This is precisely the role of the loop $\mathbf{O}$, representing the 'self' in the experience information state, $<======\mathbf{O}$, Kant's deeply perceptive insight is realized in the model. A suggestion for the neural process involved in such a synthesis is given below. It shows that Kant's argument in favor of the existence of the self, which he developed to counter David Hume's questions against it, can be put on a firm neurological basis. 
D3. Catastrophe Information ${ }^{33}$ : Any theory of Gestalts suggests that object recognition during perception involves matching the gestalt with a library of forms generated previously. Cognition involves recognition. Learning to recognize a new kind of object requires establishing a new kind of form in the library of forms, which is used in the matching process - rather like learning the meaning of a new word when learning to speak a language. It may take many repetitions of the word in different sentences before its meaning is correctly incorporated into the overall gestalt conveyed by the phrase or sentence containing it. But how can 'forms' be directly encoded without recourse to digital information?

Enter the great French mathematician, Rene Thom. His Catastrophe Theory ${ }^{59}$ constitutes a study of singularities generated by non-linear differential equations. Topologically, singularities of various levels of complexity, such as the 'fold catastrophe' and the 'butterfly catastrophe', can be created, each associated with objects or processes of various different kinds e.g. simple critical points for the first, and 'tricritical points' for the second. Thom's ambition was to use his singularities to show how morphogenetic processes occur during embryogenesis, but at that time little was known of epigenetic regulation; rather the function of genes as repositories of information encoding proteins dominated biology. Genes encode proteins, but being self-folded structures produced from linear chains of amino acids, proteins do not require Thom's theory to explain the emergence of their detailed.

Epigenetics, the regulation of gene expression, is different. The complexity theory of genetic networks developed by Kauffman makes the presence of Thom's catastrophe singularities inevitable in epigenetic processes. One of Noble's current principles of systems biology ${ }^{57}$ is that, 'there is no genetic program'. The corollary is that 'the supposed genetic program is an epigenetic program'. Regulation of gene expression is located at the edge of chaos; its criticality states involve higher order critical points of arbitrarily high order, embodying catastrophes of corresponding complexity. An ordinary critical point contains a fold catastrophe; a tricritical point ${ }^{14}$ contains a butterfly catastrophe, and so on. Complexity biology can incorporate catastrophe theory into an epigenetic program directing successive stages of morphogenesis.

The details of which catastrophe is used at each stage of morphogenesis such as gastrulation now requires detailed investigation; specifically, how corresponding non-equilibrium biochemical 
processes of the kind identified by Ilya Prigogine in his 'Order out of Chaos' generate them. The corollary to this realization is that complexity makes morphogenesis open to scientific theory.

D4. Criticality States as Cognitive States $^{33}$ : Identification of states of experience as criticality states automatically means that a library of forms becomes available to support gestalt cognition: the various different catastrophes contained in higher order critical points, starting with the fold catastrophe and butterfly catastrophe mentioned above. These can be modeled by networks of neurons. In the 1970's Stanley's group investigated magnetic critical points of orders three ${ }^{14}$ and four ${ }^{60}$, realizing that these were the start of a series of potentially unlimited complexity. ${ }^{61,62}$ The neural net-spin glass isomorphism ${ }^{63}$ means that each layer of the brain cortex can model critical points of similarly unlimited complexity. Networks of neurons such as those in brain cortices are therefore capable of supporting higher order critical points of any higher order, meaning that their catastrophes become available for use in the brain's library of gestalt forms.

Criticality states and their $\angle======\mathbf{O}$ information support gestalt cognition. They form the basis for a new dimension of cognitive science with gestalt cognition in the central role, and gestalts and their interrelationships playing central roles in both semantics and semiotics. This has an important secondary implication: the central process in thinking is not logic; the primary process is rather association. By that, brains naturally connect cognition through associated memories to the next course of action. That is how skills are learned, and skill sets developed.

D5. Why Networks of Neurons were Adopted for Physiological Regulation: Genetic networks are also capable of generating critical points of unlimited complexity. This suggests a reason for the adoption of neuronal networks as regulatory systems in early phyla in the animal kingdom; for example, in Nematoda, almost half the cells of C. Elegans are nerve cells. Even an organism with a simple cylindrical form like a nematode requires quite complex catastrophes for its morphogenesis. The role of a nervous system is to regulate processes in the organism's various tissues and cells. Only a neuronal network is capable of modeling the different catastrophes present in cellular genetic networks; neuronal networks are uniquely capable of regulating organisms. That would seem be the reason why life adopted them for use in regulation.

\section{DISCUSSION}


The new theory has implications for many different fields, not only phenomenology, information theory, cognitive science, and neuroscience, as discussed in the last section. One example is that it provides a definitive solution to Schrodinger's famous cat paradox. Schrodinger objected to the idea that even macroscopic entities could in principle be put in states of superposition, or as-yetundecided coexistence. He proposed his celebrated example of a cat, unobserved in a closed in a closed container, that would be killed if a hydrogen cyanide capsule was broken triggered by a random cosmic event. According to naive quantum theory, the cat would be suspended in coexisting dead and alive states until observed by an external observer, which intuitively seems unacceptable - paradoxical. Clearly the relationship between the quantum world and classical world requires better definition. In the context of the new theory of experience information, where the self is able to reduce its own wave function, a cat is a perfectly self-observing system, and would automatically go into one state or the other without the need for an external observer. The paradox is solved. Various publications by Penrose also refer to the Cat Paradox, pointing out that Orchestrated Reduction of wave packets solves the problem.

\section{Criticality States and Phenomenology 1: Embodiment and Sense Perception}

How may criticality states fulfil the fundamental points made by phenomenology? Clearly, criticality states are well recognized states in biology, with evidence of many kinds for their existence in biological control systems, particularly epigenetic cellular regulation, cell signalling systems, and the various neuronal networks of cortices in central nervous systems. This means that Merleau-Ponty's requirement of embodiment ${ }^{4}$ is automatically fulfilled. As regards his emphasis on perception ${ }^{4}$, a few words are necessary to explain how information from external perception enters such states.

In his book, 'Languages of the Brain ${ }^{64}$, Karl Pribram emphasized that when information from perception is registered in digital format of the surface of sensory cortices, like the visual cortex, it has not yet entered conscious experience. It is held in such states by a process of 'lateral inhibition', preventing it being scrambled by adjacent neuronal firing sequences. To transmit the sensory information to experience, cortical neurons utilize a process 'inhibition of lateral inhibition', which results in the spreading of the information all over the cortex, a linear transformation, Pribram and colleagues developed the linear transformation idea in detail ${ }^{65}$, because it explains why memories are not lost when large portions of the brain are destroyed. 
Memory seems to be retained in a form analogous to a hologram, spread all over the surface of the cortex. In holography, holograms are created by a linear transformation, the Fourier transform, which can also be used to read the hologram, because Fourier transforms are their own inverse.

Perception uses cortical information states of both kinds. For processing information transferred by the visual pathway from the retina to the visual cortex, prior to its registration in experience the brain uses digital type format reminiscent of a television, as is widely demonstrated by neuroscientists. Registration in experience is then effected by the inhibition of lateral inhibition ${ }^{64}$ linear transformation process. We propose that the ILI transform converts the information into a cortical state at criticality, the form in which it is apprehended by conscious experience.

Criticality states in brain cortices maintain entire layers of cortex at criticality, where sensory information is represented in a form like a hologram. The linear transformation integrates information pertaining to each sense. This provides the detailed explanation for both the observations of integrated information by Tononi and his colleagues ${ }^{29}$, and for Baars' Global Workspace ${ }^{28}$ with its integration of different classes of information pertaining to different senses and activitiesinto a single richly connected pattern of experience. By this mechanism, cortical criticality states informed by the action of inhibition of lateral inhibition on previously digital information embody the experience of perception. As to the question of how the self registers such information, see the discussion in the Results section above.

\section{Criticality States and Phenomenology 2: Being and Time}

A striking aspect of the experience of time is its smoothness. Information registration in conscious experience through inhibition of lateral inhibition occurs at discrete intervals, yet our experience of time seems continuous and smooth, rather than jerky. This suggests that our experience of time passing is upheld by a deeper mechanism than discrete updating of cortical information states. Clearly digital cortical information states, like those used in pre-processing, are purely static and cannot support a 'sense of time passing'. The place to look is therefore the new kind of information state with its attached loop $\angle======\mathbf{O}$. The loop with its gain $\mathbf{g}=\mathbf{1}$, is continuously circulating information, and with it the possibility of providing the basis for a smooth continuous experience of time, as Schwartz ${ }^{1}$ first suggested.

1Schwarz G. Private Communication. 
Experiences during meditation support this idea. Yoga texts declare that when all information content in the mind has been eliminated, the meditator enters a state of 'Amness' (Asmita), in which experience is purely of the sense of time passing. In the proposed model, elimination of information content corresponds to an information vector mixture is reduced to $<\mathbf{O}$, a pure loop. The criticality information state model predicts that such experience is attributable to the loop. Indeed, it is common to use the word 'Being' to describe such states, particularly when all sense of individuality has dropped away, and the meditator's experience has become purely one of impersonal 'Being', as happens beyond the 'Asmita' state. All sense of personal individuality has dropped away to leave an impersonal sense of pure existence - Absolute Being.

Furthermore, if one asks what kind of 'self' is represented by the criticality state, $<\mathbf{O}$, without information content, one can only reply ${ }^{2}$ that it is totally abstract, and beyond the possibility of qualification. No sense of individuality is attached to it, and no qualia can be ascribed to it.

Its relationship to C.S. Pierce, whose semiotics concern the interpretation of perception, is more subtle. Some may consider Pierce's phaneroscopy,or totality of all possibilities in awareness ${ }^{66}$, a significant contribution to phenomenology. However, the state of pure awareness is prior to semiosis, making interpretations of its initial experience problematic, and requiring guidance by a teacher well experienced in it. As the ground for semiosis applied to its content, it is itself devoid of semiotic activity. It cannot be constructed by semiotic means. This nullifies attempts, such as 'Cybersemiotics' ${ }^{18}$, to construct consciousness by semiotics.

Personally, reflection on my own conscious experience and ability to respond to situations and create influences I prefer in my environment, leads me to conclude that consciousness is a source of order. As conscious beings, we are able to perceive order and disorder in our environment, and where necessary create environments of our choice. We see patterns such as symmetries, and derive abstractions of such patterns as in e.g.groups and other kinds of algebra in mathematics.

In this sense, mathematics offers seemingly objective languages such as set theory or kinds of categoryother than the category of sets to describe order of different kinds, involving various kinds of relationships - sets, algebras, Calabi Yau spaces, or categories themselves. If our

2As a teacher of meditation with over 40 years experience. 
consciousness can create order, it is natural to assume that mathematics of some appropriate, possibly new, kind will be able to describe aspects of conscious experience.

Popper considered mathematics a Class 3 inter-subjective activity (between subjects), distinct from his Class 1 of objects, and class 2 of subjects. If mathematics can describe order of various kinds, it should also be able to describe the kind of order within the world of matter that leads to the possibility of Popper Class 1 and Class 2, I and Thou, subjects. However, it is not necessarily able to describe awareness itself - since that is not objective.

My knowledge of myself, I, as a subject, is subjective - by definition. In contrast my knowledge of 'Thou' as a subject is limited by the degree to which I can couple to the ' $\mathrm{I}$ ' that is you - that is

to 'Thou'. If as a Yogi I can couple directly into your heart, I can know the 'Thou' that is you more intimately than if I can only couple by speech, and other means involving external sense perception. I can then create a deeper level of connection than if I am merely limited to eyes and vision, or to ears and language.

In his review of work on phenomenological experience, Tegmark ${ }^{22}$ suggested that consciousness should be considered a special state of matter that he wittily named, 'perceptronium'. He briefly remarked, 'such an approach provides interesting links to condensed matter criticality', but did not elaborate further. We can complete his insight: Perceptronium is a state of criticality at the apex of a biological regulatory system, controlling the entire organism. With one proviso: a state of system instability, with excitations only loosely coupled to the underlying material, does not really qualify as a 'state of matter'; more appropriate would be to call it a 'state of mind'.

\section{Mind versus Matter}

In my personal experience mind and matter seem completely different. So much so that I feel genuinely surprised when scientific colleagues disagree. Matter is characterized by permanence of form, or in the case of a gas, of some aspect of its form, such as its density. In contrast, Mind seems to me to be characterized by extreme flexibility; by the ability to adapt almost instantly to any situation that arises, and make the most of new opportunities on offer. When it adapts its direction to take advantage of a new opportunity, mind stands poised in a state of internal equilibrium, ready to move in whatever direction is most appropriate - like a tennis player preparing to receive a service. 
The physics of permanence of form i.e. matter requires the presence of stabilizing physical oscillations - describable by quantum fields. However, the physics of mental adaptability requires something completely different. If, as suggested here, the system is at the edge of chaos, it can take advantage of local non-predictability to create the future it desires. In the case of champion tennis players, service returns can be hit in whichever direction they conceive. Mind has the ability to create an idea, transmit it to the motor cortex, and put it into action. Tell the CEO of any Fortune 500 company that such is not the case, and see the reaction you provoke!

Quite how the six layered structure of the neocortex permits the conception of an action originating in the brain to be set in motion in the outside world is beyond the scope of the present article, but it is no more difficult a problem to solve than the nature of dual aspect information in experience. It just requires identifying available freedoms and playing with them until a reasonable sequence of processes is identified.

Suffice it to say that the quantum nature of reality contains the possibility that the conscious creative intelligence inherent in experience can inject information into the world of experience when it chooses to do so, using Penrose's OR, not in sense perception, but in control of action. An appropriate model of the world of sense perception is required: a quantum world unfolding in time through the injection of information produced in quantum wave packet reduction events, as Stapp and others consider correct. In this picture of reality, conscious beings can be coparticipators in the unfolding of creation, by orchestrating the reduction of wave-packets within their own motor cortices.

Mind and Matter: Granted that matter ordinarily presents us with stable properties, while a healthy mind offers extreme adaptability (based no doubt on stable personality characteristics like self-esteem and self-confidence), it is natural to describe the former by properties stabilized by quantum fields, and the latter by critical instabilities where the stabilizing quantum fields have been annihilated - by processes of self-observation. Minds are criticality based, singular self-observing systems.

Descartes was at pains to point out that mind, Res Cogitans, and matter, Res Extensa, are completely different. Again, my personal view is in agreement, categorically mind and matter are in complete contrast to each other, suggesting that different physics should be employed to 
describe them. That the physics of instability is totally different from the physics of stability therefore affords me considerable satisfaction, though I recognize that this may only be a personal view.

If we accept this position that the essential difference between mind and matter requires equally different physical descriptions, a quick way of identifying the physics of mind is available. Mind, if it exists is apparently able to control matter. If I choose to raise my arm I can do so. If it was holding a tennis ball which got tossed in the air, I may be able to hit an appropriate service.

By implication, control theory is the natural candidate to look for a place to find physics different from the quantum physics of stability characteristic of matter. But the only conditions under which this obtains is at feedback instabilities, the mathematical singularities identified by Wiener who makes the specific point that his integro-differential equations, and the feedback singularities contained in them make Cybernetics, his mathematics and physics of control, completely different from all previous physics based on ordinary partial differential equations. In this light, the instabilities in control theory ${ }^{50}$, should have been the natural first candidates to explore in attempting to develop a physical theory of mind and its decision making processes.

This article has told the story of how such a theory unfolds, and how an ability to support a sense of identity and agency, with a 'sense of self', a sense of 'being in time', and a 'sense of presence' emerges from it. These properties may also help explain why criticality states apparently play a universal role at the locus of control of biological organisms. Possessing an awareness of time passing, being in time, and a sense of agency based on a sense of self, will greatly increase an organism's effectiveness in making appropriate decisions in complex, changing environments, i.e. its 'street-cred'.

As regards the main results based on long-range coherence, systems identified by material science with long-range coherence encompassing the entire system are all instabilities. Longrange, all-inclusive coherence seems to be a form of internal organization strictly alternative to quantization. Systems possessing it have singular potentials. Those supporting quanta as systems of stabilizing normal modes possess Hooke's Law potentials. Normal modes are requisite for stability of form, the fundamental of matter. Edge of chaos instability is required for an entity to make choices. It should be taken as a fundamental of mind. So also should non-linearity, the 
essence of which is a mathematical singularity representing physical instability, at the root of the singular self-observing systems at the foundation of phenomenal experience.

\section{SUMMARY AND CONCLUSION}

With their dual aspect structure, $<=====\mathbf{O}$, information states at criticality include the 'sense of self' inherent in all experience, and can support the internal sense of the passing of time that pervades experience, and, in $<\mathbf{O}$, Pure Being. They constitute authentic representations of experience, satisfying all scientific prerequisites for a theory of experience.As a model of phenomenal experience based in complexity biology, they fulfill all proposed scientific and philosophical requirements. The model has implications for many scientific fields; experts in each field should search for possible conflicts or internal inconsistencies, to see where it may need further refining or extending.

\section{ACKNOWLEDGEMENTS}

I should like to acknowledge many conversations over the years with Madan Thangavelu, and much encouragement from Brian Josephson. Helpful insights from Stuart Hameroff and Gary Schwarz are acknowledged, and discussions with Madhu Thangavelu, Judu Ilavarasu, ECG (George) Sudarshan, and Steven Weinberg. Finally, I am particularly grateful to Arran Gare for helpful advice, and to the editor of the Special Issue for his constant encouragement, advice, and the trust he has shown in my presumed ability to deliver. 


\section{REFERENCES}

1. Brentano F. Descriptive Psychology, transl. by Benito Müller, London: Routledge, 1995.

2. Husserl E. The Phenomenology of Internal Time Consciousness. Indiana University Press, Bloomington, 1964.

3. Heidegger M. 1927. Sein und Zeit (Tübingen): Niemeyer. 19. Aufl. (2006), ISBN-10: 3484701536; ISBN-13: 978-3484701533. Being and Time. Translated by John Macquarrie and Edward Robinson. New York: Harper and Row, 1962. Harper Perennial Modern Thought edition (2008). ISBN-10: 0061575593; ISBN-13: 978-0061575594..

4. Merleau-Ponty, M. 1945. Phénoménologie de la perception. (Paris): Gallimard. Phenomenology of Perception. (Colin Smith tr.) Routledge \& Kegan Paul, London, 1962. Routledge (2013).

5. McCullough W. Pittts W. A Logical Calculus of the Ideas Immanent in Nervous Activity. Bulletin of Mathematical Biophysics, 1943; 5: 115-133.

6. Chalmers D. The Conscious Mind. Oxford University Press, Oxford, 1997.

7. Shear J. (Ed.) Explaining Consciousness: the Hard Problem. Academic Press, London, 1997.

8. Anthony L. Spence G. The Elephant Whisperer: Learning about Life, Loyalty, and Freedom from a remarkable Herd of Elephants. Pan Books, London, 2010.

9. Adamson J. Born Free. Harvill Press, New York, 1960.

10. Nagel. T. What is it like to be a bat? Philosoph Rev, 1974; 83:435-450.

11. Penrose R. The Emperor's New Mind: concerning computers, minds and the laws of physics. Oxford University Press, Oxford, 1999.

12. Kauffman S.A. Gare A. Beyond Descartes ad Newton. This issue p.?

13. Schroeder M.J. Vallverdu J. Situated Phenomenology and Biological Systems: Eastern and Western Synthesis. This issue p.?

14. Hankey A. Stanley H.E. Chang T.S. Geometric Predictions of Scaling at Tricritical Points. Phys Rev. Lets. 29(5); 278-281. (1972).

15. Hankey A. Note on Nonlinear Realizations of Conformal Symmetry. Phys Rev D3(10), 2543-2546 (1971).

16. Godine J. Hankey A. Effects of a Neutral Weak Current in the Process $e^{+} e^{-} \rightarrow \mu^{+} \mu$. Phys. Rev. D 6, 3301-3306 (1972). 
17. Hagelin S. Parker R.J.D. Hankey A. Does weakly interacting dark matter change the primordial abundance of light elements? Physics Letters B 215(2): 397-403 (1988).

18. Brier S. Cybersemiotics: a new foundation for transdisciplinary theory of consciousness, cognition, meaning and communication. Biosemiotic Origins of Mind. Springer 2012.

19. Josephson B.D. The elusivity of nature and the mind-matter problem. Arxiv.1409.2168.

20. Penrose R. Shadows of the Mind: A search for the missing science of consciousness. Oxford University Press, Oxford, 1994.

21. Hameroff S. Penrose R. 'Consciousness in the universe: a review of the 'Orch OR theory' Phys Life Rev, 2014; 11(1):39-78.

22. Tegmark M. Consciousness as a state of matter. arXiv:1405.0493v1 [physics.pop-ph] 2 May 2014.

23. Shear J. The Inner Dimension: Philosophy and the Experience of Consciousness. New York: Peter Lang (1990).

24. Travis F. Shear J. Focused attention, open monitoring and automatic self-transcending: Categories to organize meditations from Vedic, Buddhist and Chinese traditions. Consciousness and Cognition, 2010; : .

25. Varela F. Shear J. (Eds.) The View from Within. Thorverton: Imprint Academic (1999)..

26. Damasio A. Self Comes to Mind: Constructing the Conscious Brain. Vintage, USA, 2012.

27. Chalmers D. 'Facing up to the Problem of Consciousness.' J. Consc. Studies, 1995; 2(3):200-219.

28. Baars B. In the Theatre of Consciousness: The Workspace of the Mind, NY: Oxford University Press, 1997, ISBN 0-19-5114703-0.

29. Tononi G. Consciousness as Integrated Information: a provisional manifesto. The Biological Bulletin, 2008; 215: 216-242.

30. Kant I. The Critique of Pure Reason. Pluhar W.S. (trans), Hackett, 1996.

31. Domash L. Physics of Coherent States. MIU Press, Rheinweiler, 1975.

32. Crick F, Koch C. A framework for consciousness. Nature Neuroscience (2003) 6:119-26.

33. Hankey A. Complexity-biology based information structures can explain subjectivity, objective reduction of wave-packets, and non-computability Cosmos and History: the Journal of Natural and Social Philosophy (2014) 10: 237-250.. 
34. Hankey A Ilavarasu J. Proceedings of Consciousness Conference, NIAS, Bangalore, 2012.

35. Tetzlaff C. Okujeni S. Egert U. Wörgötter F. Butz M. Self-Organized Criticality in Developing Neuronal Networks. PLOS, Computational Biology, The Immunology Collection, 2010. doi: 10.1371/journal.pcbi.1001013.

36. Nytker M. Price N.D. Larjo A. Aho T. Kauffman S.A. Yli-Harja O. Shmulevich I. Critical Networks Exhibit Maximal Information Diversity in Structure Dynamics Relationships. Phys Rev Lett, 2008; 100: 058702.

37. Hankey A. A new approach to biology and medicine: an expanded role for regulation. To be published. J Med S July, 2015;7:13-18.

38. Varela, F. J., Thompson, E. and Rosch, E. The Embodied Mind: Cognitive Science and Human Experience. Cambridge, Mass: MIT Press (1991).

39. Kauffman S. At Home in the Universe. The search for the laws self organization and complexity. Oxford: Oxford University Press (1995).

40. Bak P. Tang C. Wiesenfeld K. Self-organized criticality: An explanation of the $1 / f$ noise. Phys. Rev. Lett. (1987), 59, 381.

41. Bak, P. How Nature Works - The Science of Self-Organized Criticality. New York, N.Y.: Springer Verlag (1996).

42. Holland J.H. Complex Adaptive Systems. Daedalus, 1992; 121: 17-30.

43. Nurse P. Complexity and Biology. Cell, 2014; 157:272.

44. Jensen H.J. Self-organized Criticality: Emergent Complex Behavior in Physical and Biological Systems (Cambridge Lecture Notes in Physics). Cambridge University Press, Cambridge, 1995.

45. Massobio P. Et al. Criticality as a signature of healthy neural systems. Frontiers in Neuroscience, 2015; 9:22.

46. Uhlig M. Levina A. Geisel T. Hermann M.J. Self-organized criticality in structured neural networks. BMC Neurosci, 2013; 14(suppl):168.

47. Yang H. Et al. Peak variability and optimal performance in cortical networks at criticality. Chap 15 in Criticality in Neural Systems. Wiley, 2014. DOI: $10.1002 / 9783527651009$. 
48. Stanley H.E. Phase Transitions and Critical Phenomena. Oxford University Press, Oxford, 1972.

49. Mattson M.P. Hormesis defined. Ageing Res Rev, 2008; 7:1-7.

50. Wiener N. Cybernetics of control and communication in the animal and the machine. M.I.T. Press, 1948.

51. Nurse P. Life, logic and information. Nature, 2008; 454:424-426.

52. Hankey A. Studies of advanced stages of meditation in the Tibetan Buddhist and Vedic

Traditions I: A comparison of general changes. Evid Based Complement Alternat Med. 2006; $3(2), 513-521$.

53. Bassingthwaighte JB. Liebovitch LS. West BJ. Fractal Physiology. Oxford University Press 1994.

54. Brillouin L. Science and Information Theory. Dover, Mineola, NY, 1956.

55. Ho M-W. The Rainbow and the Worm World Scientific, Singapore, 1980.

56. Deutsch D. Quantum theory, the Church-Turing principle and the universal quantum computer. Pro Roy Soc A (1985) 400 (1818): pp. 97-117. Doi:10.1098/rspa.1985.007.

57. Noble D. Principles of systems biology from a physiologist's perspective. Proceedings of the Beijing Joint Conference on Physiological Sciences, 2008.

58. Shannon C.E. A mathematical theory of communication. Bell System Technical Journal, $1948 ; 27: 379-423 \& 623-656$.

59. Arnold V.I. Catastrophe Theory, 3rd ed. Berlin: Springer-Verlag, 1992.

60. Harbus F. Hankey A. Stanley H.E. Chang T.S. System Exhibiting a Critical Point of Order Four: Ising Planes with Variable Interplanar Interactions. Physical Review B 8(5): 22732278 (1973).

61. Chang T.S. Hankey A. Stanley H.E. Generalized Scaling Hypothesis in Multicomponent Systems. I. Classification of Critical Points by Order and Scaling at Tricritical Points. Phys. Rev. B 8, 346-364 (1973). DOI: 10.1103/PhysRevB.8.346

62. Hankey A. Chang T.S. Stanley H.E. Generalized Scaling Hypothesis in Multicomponent Systems. II. Scaling Hypothesis at Critical Points of Arbitrary Order. Phys. Rev. B 8, 1178-1184 (1973).

63. Bovier A. Picco P. (Eds.) Mathematical aspects of spin glasses and neural networks. Birkhauser, Boston. 1998. 
64. Pribram K. Languages of the Brain: Experimental paradoxes and Principles in Neuropsychology. Oxford, England, Prentice-Hall, 1971.

65. Pribram K. in Wilbur K. (Ed.) The Holographic Paradigm and other paradoxes: exploring the leading edge of science. Shambala, 1982.

66. Pierce C.S.: The Collected Papers of Charles Sanders Peirce. Volume 1 p. 289 Intelex CD-ROM edition (1994), reproducing Vols. I-VI, Hartshorne, C. and Weiss, P., Eds.; Harvard University Press: Cambridge, MA, USA, 1931-1935.

67. Simeonov, P. L., Matsuno, K., Root-Bernstein, R. S. (Eds.) 2013. Can Biology Create a Profoundly New Mathematics and Computation? Special Theme Issue on Integral Biomathics. Journal Progress of Biophysics and Molecular Biology. 2013, 113(1). Elsevier. ISSN: 00796107.

68. Whitehead A.N. Process and Reality: an essay in cosmology. New York: Macmillan Company, 1929. 


\section{TABLE 1: CRITICAL INSTABILITIES AND CRITICAL FEEDBACK INSTABILITIES: \\ Ten Properties}

\begin{tabular}{|l|l|l|}
\hline No. & PROPERTY & APPLICATION \\
\hline $\mathbf{1 .}$ & Restoring potentials not Hooke's Law & Not quantizable; no stabilizing quantum fields \\
\hline $\mathbf{2 .}$ & Long-range correlations & High temperature coherence \\
\hline $\mathbf{3 .}$ & Negative Entropy & Inherent Order / Low entropy of life \\
\hline $\mathbf{4 .}$ & States not separable & Fully Non-Reductive Physics \\
\hline $\mathbf{5 .}$ & Fundamental states are Mixtures & Inherent information \\
\hline $\mathbf{6 .}$ & Each Mixture carries a loop & Represent by $<=======\mathbf{O}$ \\
\hline $\mathbf{7 .}$ & Feedback loop has $\mathbf{g}=1$ & Singular self-observing system - SSOS \\
\hline $\mathbf{8 .}$ & Self-observation & Self-knowledge - Attribute of Consciousness \\
\hline 9. & Self-observation annihilates Quanta & Absence of Quanta - ORof Wave Packet \\
\hline $\mathbf{1 0 .}$ & Edge of Chaos - bifurcation region & Genuine choice possible \\
\hline
\end{tabular}

